\title{
Impact of Kernel-PCA on Different Features for Person Re-Identification
}

\author{
Md Kamal Uddin, Amran Bhuiyan, Mahmudul Hasan
}

\begin{abstract}
In the driving field of computer vision, re-identification of an individual in a camera network is very challenging task. Existing methods mainly focus on strategies based on feature learning, which provide feature space and force the same person to be closer than separate individuals. These methods rely to a large extent on high-dimensional feature vectors to achieve high re-identification accuracy. Due to computational cost and efficiency, they are difficult to achieve in practical applications. We comprehensively analyzed the effect of kernel-based principal component analysis (PCA) on some existing high-dimensional person re-identification feature extractors to solve these problems. We initially formulate a kernel function on the extracted features and then apply PCA, significantly reducing the feature dimension. After that, we have proved that the kernel is very effective on different state-of-the-art high-dimensional feature descriptors. Finally, a thorough experimental evaluation of the reference person re-identification data set determined that the prediction method was significantly superior to more advanced techniques and computationally feasible.
\end{abstract}

Keywords: Feature Dimension, Kernel-PCA, Re-identification, Video surveillance.

\section{INTRODUCTION}

$\mathrm{T}$ he computer vision community has drawn a great deal of attention in the last few years due to its significant role in visual surveillance systems, including person retrieval and tracking across disjoint cameras. The task of the Person Re-identification (Re-id) system is to retrieve the same individuals over a set of non-overlapping cameras. Despite the arduous efforts, it remains a complicated task for the nonrigid formation of the human body; a pedestrian can be observed with different perceptions and highly varying illumination circumstances, as shown in Fig. 1. To focus on these encounters, Re-identification researchers accept a common assumption that persons are not allowed to change their clothes, so their appearance in all the views is similar, at least for a short period. It will enable the researchers to

Manuscript received on August 26, 2021.

Revised Manuscript received on September 20, 2021.

Manuscript published on September 30, 2021.

* Correspondence Author

Md Kamal Uddin*, Department. of Computer Science and Telecommunication Engineering, Noakhali Science and Technology University, Noakhali, Bangladesh. Email: kamal.uddin.nstu@gmail.com

Amran Bhuiyan, Department. of Computer Science and Telecommunication Engineering, Noakhali Science and Technology University, Noakhali, Bangladesh. Email: amran.apece@gmail.com

Mahmudul Hasan, Department. of Computer Science and Engineering, Comilla University, Comilla, Bangladesh. Email: mhasanraju@gmail.com

(C) The Authors. Published by Blue Eyes Intelligence Engineering and Sciences Publication (BEIESP). This is an open access article under the CC BY-NC-ND license (http://creativecommons.org/licenses/by-nc-nd/4.0/) consider exclusively the visible aspect of people, commonly known as appearance-based methods. Furthermore, appearance-based Re-id algorithms are roughly categorized into two classes: direct method and learning-based method. A dataset of various individuals is used to discover the features and the metric space to compare them to assure a high re-identification rate in the former group. On the other hand, direct methods are primarily dedicated to searching for the most discriminant features and their combination to design a robust descriptor (or signature) for everyone.

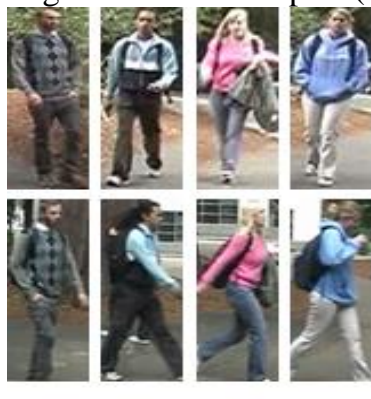

(a) VIPeR

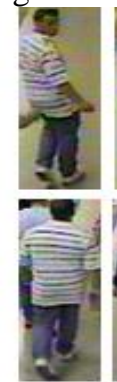

(b) GRID
Fig. 1. Example of viewpoint and illumination variations of pedestrians. (a) Sample images are taken from ViPER dataset [1]; and (b) GRID dataset [2].

Moreover, re-identification algorithms can also be grouped into multiple-shot and single-shot classes of methods. Here, the previous aims on associating pairs of images for every individual. At the same time, the final employs numerous images of a similar person as the probe and/or in the gallery set, trying to utilize this supplementary information. The direct methods emphasize designing unique features for capturing the most distinctive facets of an individual. Some state-of-the-art learning-based approaches learn a projection metric from a high-dimensional input space $[10,11]$. At the same time, learning-based approaches use the training data to learn metric spaces were to compare pedestrians to guarantee a high re-identification rate. The importance of high dimensional data makes another step forward by bridging with computational complexity. We projected high dimensional Re-id features, i.e., LOMO [12] and GOG [13] into low dimensional space. We also made those features computationally inexpensive, reduced the noise and solve the curse of dimensionality problem. Thus, our proposed approach makes the following contributions:

1) We formulate a kernel-based Principal Component Analysis (KPCA) based person re-identification method that directly projects high dimensional data into their low dimensional feature space perspective.

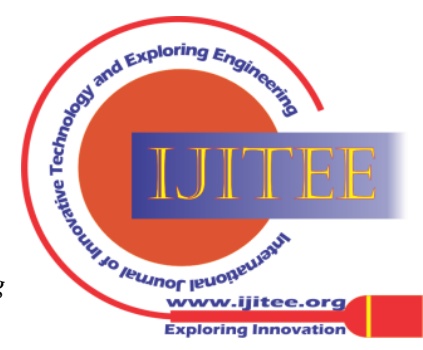




\section{Impact of Kernel-PCA on Different Features for Person Re-Identification}

2) Extensive experimental analysis is validating the benefits of our method over present options on several standard datasets. In addition to that, the analyzed KPCA is general and can be used to reduce any feature dimension.

We organized the rest of the paper as follows. We tried to find out the very recent research and reviewed these in the section II. Our proposed method is elaborately described in section III. Section IV shows the experimental results with prior efficacy. Finally, in the conclusion section V, we sum up the experiment and briefly described our views.

\section{LITERATURE REVIEW}

We have gone through many recent studies of person re-identification. Here discussed some related works, which are the Re-id method based on appearance. The most common appearance-based Reid method is the direct and learning-based method. Most direct methods are based on local characteristics, segmenting people into horizontal stripes [3], decomposable triangles [4], and then segmenting Features (SDALF), which models human appearance through symmetric and asymmetric features. In the same process, [7] proposed a Re-id method based on a custom graphical well-positioned body parts and manually weights these parts according to their prominence. Following the same idea, [8] designed a method that can automatically assign weights to body parts based on the discriminative power of body parts. In [9], a light-invariant feature descriptor based on the that single track color performs relatively well in identifying learning-based method uses the training data to learn the metric space to compare the technology of pedestrians to ensure a high re-recognition rate. The common assumption is that the information learned from the training data can be generalized to invisible probes. The method proposed in [1] uses reinforcement to select a combination of color and spatial information to achieve viewpoint invariance. Similarly, in [14], a high-dimensional signature composed of multiple features is projected into the low-dimensional discriminant latent space by partial least squares (PLS). Re-identification is presented as a binary classification problem (one-to-two). all) through [15] using Haar-like features and part-based MPEG7 dominant color descriptors, and in [16], it is presented as a relative classification problem in a high-dimensional feature space, in which true and false matches become more separable. Paired Constrained Component Analysis (PCCA) [11] and Relaxed Paired Metric Learning (RPML) [10] learn projection metrics from high-dimensional input space to low-dimensional space, where the distance between pairs of data points meets the required constraints. In [17], a statistical reasoning perspective is used to learn metrics that satisfy the equivalence limit. In [12], local feature level occurrence maximization (LOMO) has been used as a feature representation and cross-view quadratic discriminant analysis (XQDA) metric learning technique, which is an extended version of the learning metric proposed in [17], used for matching. In [13], the hierarchical use of Gaussian Embedding (GOG) of pixel features was explored. In contrast them into regions [5]. In [6], the author used a complex appearance model, Symmetric Accumulation of Local structure (CPS), which combines characteristics of several logarithmic chromaticity color space was proposed. It shows people in significantly changing image conditions. The

to the above methods, we analyze the impact of kernel-PCA on different high-dimensional features for person re-identification.

\section{PROPOSED APPROACH}

This section describes our proposed KPCA based person re-identification approach. A person's appearance under different cameras varied due to the poses and viewpoints variations. However, appearance features lie on input space when different well known feature descriptors, such as LOMO [12], WHOS [20], SDALF [21], CPS [7] and ELF [1], are used to extract features. KPCA can map the input space into higher-dimensional nonlinear space which finally reduced to lower-dimensional common subspace, as shown in Fig. 2. Consider a dataset, captured from camera "A" or camera "B", though it can be more than two cameras in a camera network. First, we extract features of each image using LOMO, WHOS, SDALF, CPS and ELF feature descriptors, where images are taken from an individual captured by different cameras which are installed in different disjoint locations. Each image feature is represented as $z_{i}$ with l-dimensional vector, where $i=1,2,3, \ldots, \mathrm{N}$. Then we construct Kernel matrix by considering a nonlinear transformation which converts the original l-dimensional feature space into an $m$-dimensional feature space, where each data point $z_{i}$ is projected to a point $\phi\left(z_{i}\right)$ with high-dimensional feature space. Though we can obtain the new feature space by performing standard PCA, but this can be extremely costly and inefficient. So, we use kernel methods to simplify the computation [18]. The kernel methods cannot compute $\phi\left(z_{i}\right)$ explicitly. Therefore, we compute the kernel matrix of the feature vectors $\left\{z_{i}\right\}$ using the Gaussian kernel [19],

$$
k(z, y)=\exp \left(-\frac{\|z-y\|^{2}}{2 \sigma^{2}}\right)
$$

with parameter $\boldsymbol{\sigma}$.

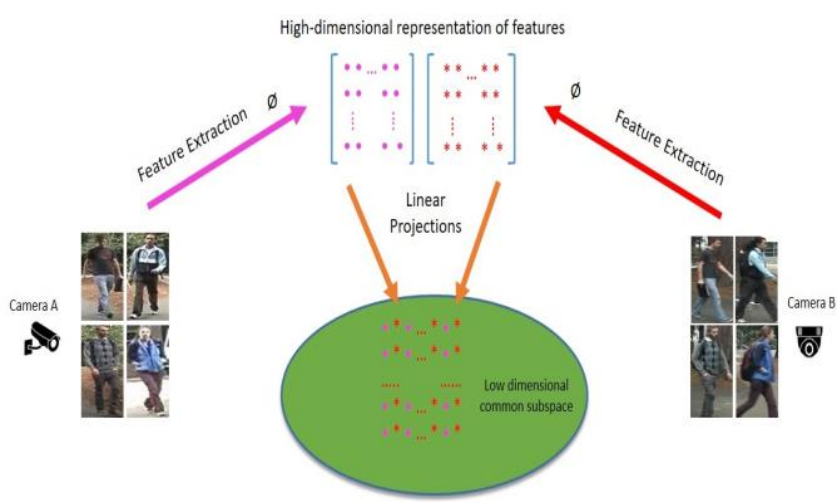

Fig. 2. KPCA based person re-identification approach.

\section{EXPERIMENTS}

In this section, we evaluate the performance of our approach by performing experiments on the benchmark re-identification datasets using Kernel-PCA. First, we use five novel person re-identification feature descriptors which are LOMO [12], WHOS [20], SDALF [21], CPS [7] and ELF [1], to extract features from those datasets.

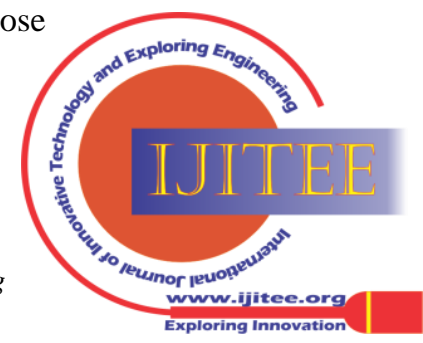


Second, we apply Gaussian kernel on extracted features then apply PCA on that feature which can effectively reduce the feature dimension which dramatically reduces computational time for next steps. Finally, we report the results.

\section{A. Datasets}

There are several freely accessible Re-id datasets, where some of which are considered for our experiments. The benchmark datasets we consider here for our experiments are VIPeR [1], CAVIAR [7], and WARD [22]. Each dataset has specific characteristics such as the number of instances per subject, the number of subjects considered, pose and illumination differences, the severity of occlusions, the number of viewpoints, and the captured image resolution. Re-id can be separated into single-shot and multi-shot modalities based on the number of occurrences of a subject in the dataset. In case of a single shot, one for the probe and one for the gallery. For multi-shot, there are multiple instances per subject, which help extract the subject's vital information. The attributes of those datasets are as follows:

VIPeR Dataset [1] encompasses 632 pedestrians with two views. The same pedestrians are considered for capturing the same pair of data from arbitrary viewpoints under varying illumination conditions. Every image size is equal and is 128 $\times 48$ pixels, presenting a focused, unoccluded human figure.

WARD Dataset [22] contains 4,786 pictures of 70 diverse individuals obtained by three non-overlapping cameras in a real-world reconnaissance situation. This dataset is of specific intrigued since it includes an enormous brightening variety separated from determination and postures alter. We conduct the tests for all the three diverse cam-era sets, indicated here as camera sets 1-2, 1-3, and 2-3.

CAVIAR4REID Dataset [7] contains pictures of people on foot disengaged from the CAVIAR store [7], giving a challenging real-world setup. This is often the freely accessible dataset where the intra-person pictures change a parcel in terms of resolution, light, posture, etc. The biggest challenges in this dataset are its wide changes in determination, as the pictures' least and most extreme sizes are $17 \times 39$ and $72 \times 144$, separately. Radical posture varieties make it more challenging. From 72 recognized diverse people, 50 are captured by two cameras with 20 pictures for each of them and 22 from one camera, with 10 ten pictures for each of them. Here we limited the subjects taken from 2 cameras and chose 5 five irregular pictures from the primary camera for the test set and five arbitrary pictures from the moment camera as exhibition set. At that point, we performed multi-shot re-identification. All pictures are resized to $32 \times 96$ pixels.

\section{B. Performance Analysis}

We report the results in terms of recognition rate. Most of the existing person re-identification methods use cumulative matching characteristic (CMC) curve as performance evaluator [7], [8], [20], [21], [12], [23] where it considers the correct match in the top $m$ matches. The performance can also be evaluated by calculating the ranked matching rates. Higher the ranks value the better the Re-id approaches.

\section{Re-identification by applying Kernel-PCA Technique}

In this section, we perform the experiments to compare the performance of our proposed Kernel-PCA based approach with some state-of-the-art extraction methods including LOMO [12], WHOS [20], CPS [7], SDALF [21] and ELF [1].

Implementation Details. In our all experiments, we use publicly available codes of LOMO [12], WHOS [20], CPS [7], SDALF [21] and ELF [1] and tested our approach on experimental datasets. After applying our proposed kernel trick on the LOMO, WHOS, CPS, SDALF and ELF, we refer the features as K-LOMO, K-WHOS, K-CPS, K-SDALF and K-ELF, respectively. In our experiment, we consider only HSV histogram features for SDALF and CPS.

Mention that, we use the standard dataset splitting to make the comparison fair.

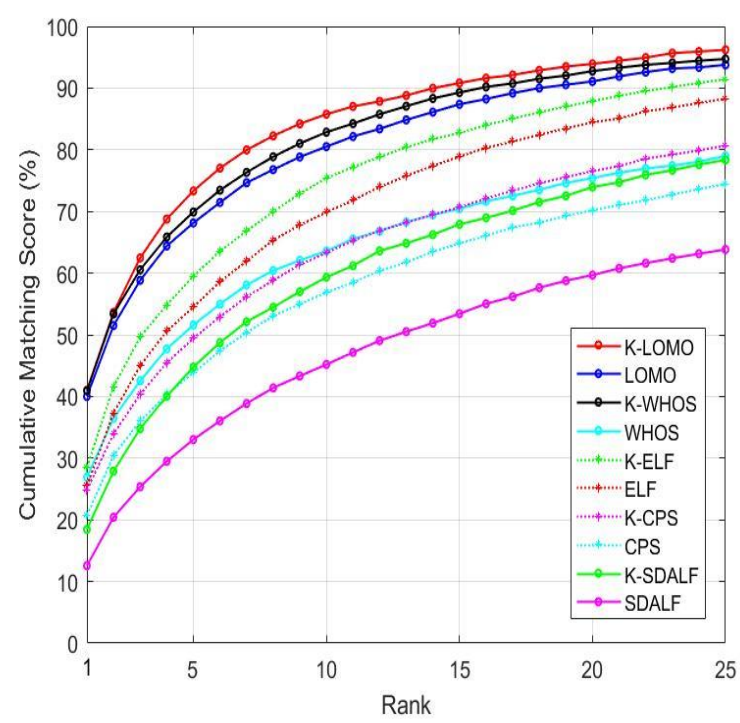

Fig. 3. Comparison of our approach with some state-of-the-art methods: LOMO, WHOS, CPS, SDALF and ELF for VIPeR dataset.

Results. Fig. 3 shows the comparison of our proposed approach with the state-of-the-art methods for VIPeR dataset. Table I and II reports the results for CAVIAR4REID and WARD datasets. From these tables and figure, the following observations can be made: (i) for rank-1, overall recognition accuracies of our proposed Kernel-PCA based approach for person re-identification is better than all compared methods on all the experimental datasets. (ii) rank-1 performance improvement over LOMO, WHOS, ELE, CPS and SDALF are $1.01 \%, 14.02 \%, 2.97 \%, 4.17 \%$ and $5.89 \%$ respectively on VIPeR dataset. (iii) For CAVIAR4REID dataset, it is 2.8\%, $13.6 \%, 1.6 \%, 3.2 \%$ and $5.2 \%$ respectively. (iv) Table II shows the results for WARD dataset where rank-1 performance improvement over LOMO, WHOS, ELF and SDALF are $6.67 \%, 9.53,1.91 \%$ and $3.81 \%$ respectively, only CPS fails to improve the performance.

Published By:

Blue Eyes Intelligence Engineering and Sciences Publication

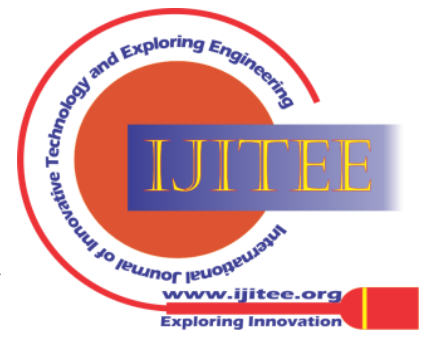




\section{Impact of Kernel-PCA on Different Features for Person Re-Identification}

Table-I. Comparison of our methods with some existing state-of-the-art methods for CAVIAR4REID dataset reported by top-ranked matching rate (\%).

\begin{tabular}{|l|c|c|c|c|c|}
\hline Methods & Rank 1 & Rank 5 & Rank 10 & $\begin{array}{c}\text { Rank } \\
15\end{array}$ & $\begin{array}{c}\text { Rank } \\
20\end{array}$ \\
\hline CPS [7] & 10 & 43.6 & 70.4 & 84.4 & 94.8 \\
\hline K-CPS (Ours) & $\mathbf{1 3 . 2}$ & 46.8 & 69.6 & 86.8 & 96.8 \\
\hline ELF [1] & 14.8 & 54 & 76.8 & 93.6 & 99.6 \\
\hline K-ELS (Ours) & $\mathbf{1 6 . 4}$ & 50.8 & 78.8 & 93.6 & 99.2 \\
\hline SDALF [6] & 14 & 52.8 & 72.4 & 80.8 & 97.6 \\
\hline $\begin{array}{l}\text { K-SDALF } \\
\text { (Ours) }\end{array}$ & $\mathbf{1 9 . 2}$ & 58 & 80.8 & 94.8 & 99.2 \\
\hline WHOS [15] & 16 & 58.8 & 84.4 & 94 & 98.8 \\
\hline $\begin{array}{l}\text { K-WHOS } \\
\text { (Ours) }\end{array}$ & $\mathbf{2 9 . 6}$ & 66.8 & 85.6 & 94.8 & 97.6 \\
\hline LOMO [24] & 28.8 & 73.2 & 90.8 & 96.8 & 100 \\
\hline $\begin{array}{l}\text { K-LOMO } \\
\text { (Ours) }\end{array}$ & $\mathbf{3 1 . 6}$ & 68.8 & 86.8 & 95.2 & 99.2 \\
\hline
\end{tabular}

Table-II. Comparison of our approach with existing methods for WARD dataset.

\section{Effect of Parameter Variations} approach for person re-identification by varying the feature dimension $n$ and the value of $\sigma$ in (1).

Implementation Details. Kernel-PCA directly determines the performance of our proposed method. When kernel-PCA is applied on different features then eigenvectors are arranged according to descending order and first $n$ eigenvectors, also called $n$ feature dimensions, are kept. Feature dimension $n$ vary for each method to get better accuracy mostly varied from $n=50$ to 350 .

The most influential parameter for Gaussian kernel PCA is $\sigma$, which is defined in (1). The Gaussian kernel is the function of distance $\|z-y\|$ between two vectors $z$ and $y$. More specifically, if we want to distinguish the different classes in the new feature dimensional space, then the parameter $\sigma$ should be larger than intra-class distances, and smaller than inter-class distances. Moreover, if we want to estimate the intra-class or inter-class distances, then first we should know how many classes are in the whole dataset, otherwise, we have to set $\sigma$ to a small value to cover only the surrounding information of each data point. In this case, for each data point $z_{i}$, we consider the distance from $z_{i}$ to its nearest neighbor is $d_{i}^{N N}$. In our experiments, we prefer the following parameter selection technique which used in [24].

$$
\sigma=\gamma \times \operatorname{mean}_{i}\left(d_{i}{ }^{N N}\right)
$$

Where value of $\gamma$ parameter vary from 1 to 10 . In our experiments, this technique confirms that the value of $\sigma$ covers sufficient surrounding data points, where the inter-class distances are larger than the value of $\sigma$.

\begin{tabular}{|l|c|c|c|c|c|}
\hline Methods & Rank 1 & Rank 5 & $\begin{array}{c}\text { Rank } \\
10\end{array}$ & $\begin{array}{c}\text { Rank } \\
15\end{array}$ & $\begin{array}{c}\text { Rank } \\
20\end{array}$ \\
\hline CPS [7] & $\mathbf{4 0 . 5 7}$ & 74.67 & 88.95 & 93.43 & 95.81 \\
\hline K-CPS (Ours) & 34.76 & 67.24 & 84.48 & 92.86 & 96.86 \\
\hline ELF [1] & 48.57 & 80 & 90.48 & 92.38 & 94.29 \\
\hline K-ELS (Ours) & $\mathbf{5 0 . 4 8}$ & 70.48 & 91.43 & 94.29 & 97.14 \\
\hline SDALF [6] & 24.76 & 58.1 & 75.24 & 85.71 & 89.52 \\
\hline $\begin{array}{l}\text { K-SDALF } \\
\text { (Ours) }\end{array}$ & $\mathbf{2 8 . 5 7}$ & 60.95 & 82.86 & 90.48 & 96.19 \\
\hline WHOS [15] & 49.52 & 81.9 & 92.38 & 98.1 & 100 \\
\hline $\begin{array}{l}\text { K-WHOS } \\
\text { (Ours) }\end{array}$ & $\mathbf{5 9 . 0 5}$ & 85.71 & 92.38 & 98.1 & 99.05 \\
\hline LOMO [24] & 76.19 & 94.29 & 96.19 & 99.05 & 100 \\
\hline $\begin{array}{l}\text { K-LOMO } \\
\text { (Ours) }\end{array}$ & $\mathbf{8 2 . 8 6}$ & 94.29 & 96.19 & 100 & 100 \\
\hline
\end{tabular}

We further analyze the performance of kernel-PCA based

Results. Fig. 4 reports the performance of K-LOMO for VIPeR dataset on different dimension. From this figure, we observe that the dimension at 190, rank-1 recognition rate for K-LOMO was the highest.

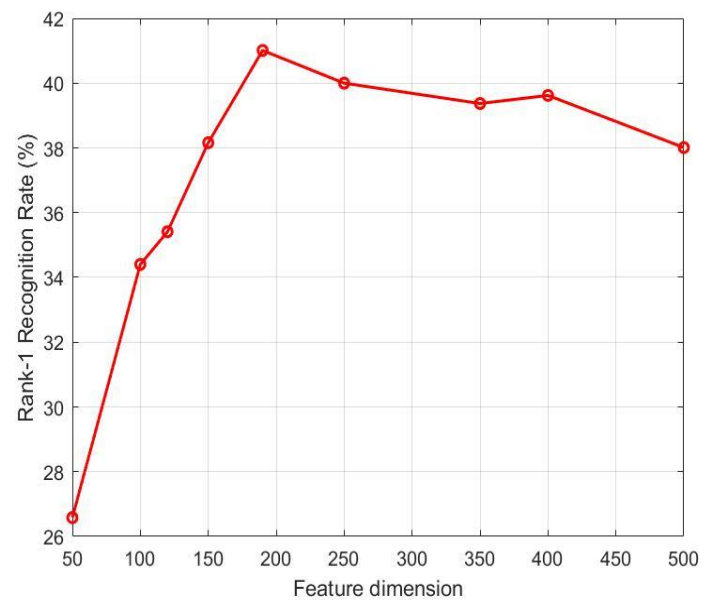

Fig. 4. Effect of dimension variation on the performance for K-LOMO on VIPeR dataset.

Fig. 5 shows the variation of gamma $(\gamma)$ value, which is directly affect the sigma value $(\sigma)$ for the performance of K-WHOS, K-ELF and K-CPS on VIPeR dataset. From this figure, the following observation can be constructed: (i) our proposed approach achieves the best results for K-WHOS, $\mathrm{K}-\mathrm{ELF}$, and K-CPS on VIPeR dataset when $\gamma$ varies around 2.0 to 3.0 .
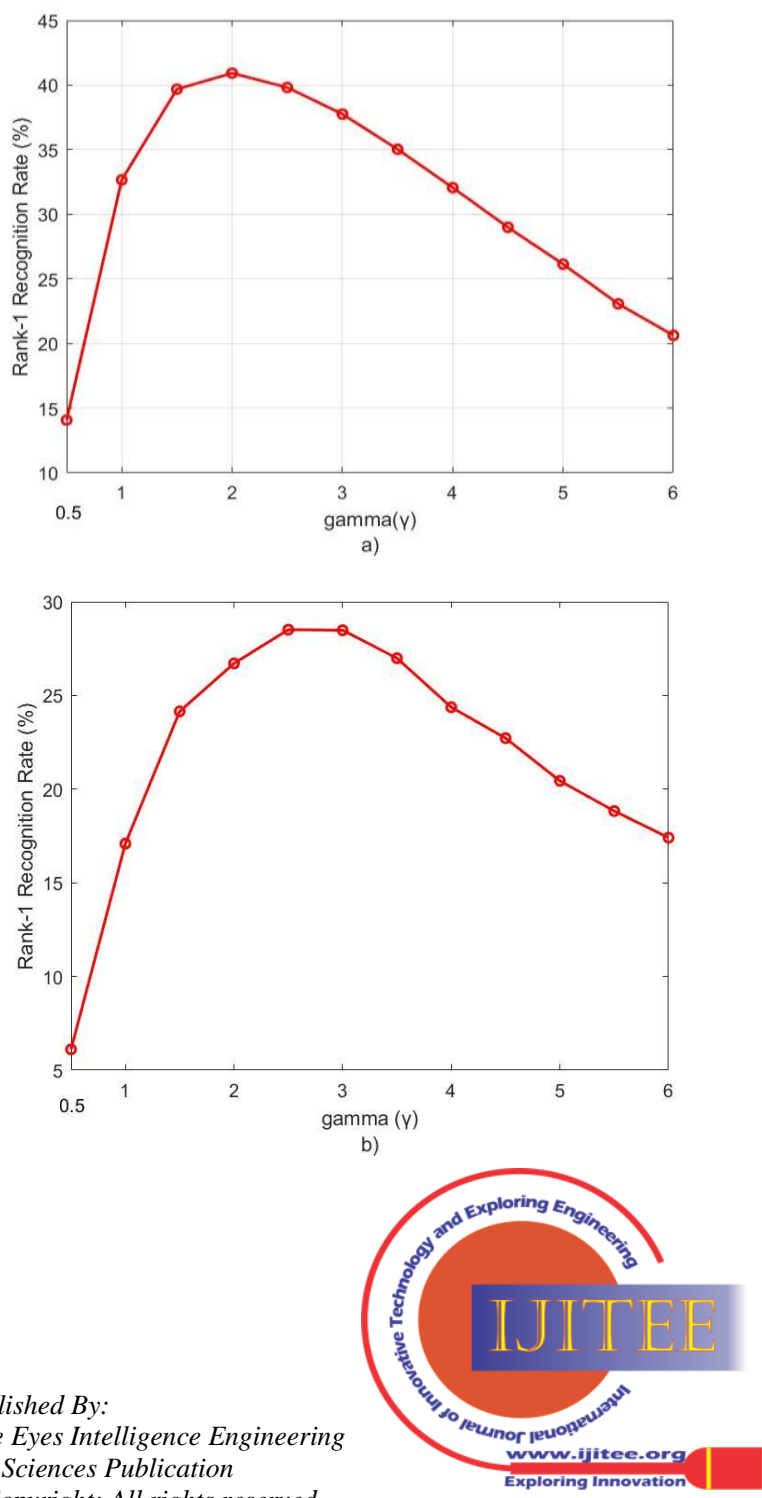


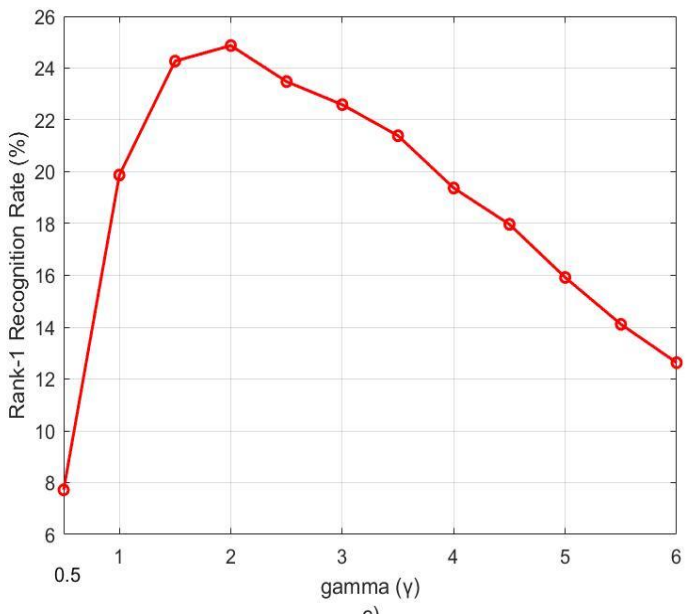

c)

Fig. 5. Plots (a-c) show the effect of gamma $\gamma$ variation on the performance for K-WHOS, K-ELF and K-CPS on VIPeR dataset.

\section{CONCLUSION}

In this research, we have taken a thorough look at the impact of KPCA on person re-identifying feature extractors for high-dimension personnel in different states, including LOMO, WHOS, CPS, ELS, and SDALF. The key component of this work is to formulate a kernel function on the extracted features and then apply PCA on the features, which can effectively reduce the dimension of the feature. The investigational findings of our intended method show the efficiency of the reference data set to re-identify people with different characteristics.

\section{REFERENCES}

1. D. Gray and H. Tao, "Viewpoint invariant pedestrian recognition with an ensemble of localized features", In European conference on computer vision, Springer, Berlin, Heidelberg, pp. 262-275, 2008.

2. C.C. Loy, T. Xiang, and S. Gong, "Time-delayed correlation analysis for multi-camera activity understanding", International Journal of Computer Vision, 90(1), pp.106-129, 2010.

3. N.D. Bird, O. Masoud, N.P. Papanikolopoulos, and A. Isaacs, "Detection of loitering individuals in public transportation areas", IEEE Transactions on intelligent transportation systems, 6(2), pp.167-177, 2005.

4. N. Gheissari, T.B. Sebastian, and R. Hartley, "Person re-identification using spatiotemporal appearance", In IEEE computer society conference on computer vision and pattern recognition, Vol. 2, pp. 1528-1535, 2006.

5. X. Wang, G. Doretto, T. Sebastian, J. Rittscher and P. Tu, "Shape and appearance context modeling", In IEEE 11th international conference on computer vision, pp. 1-8, 2007.

6. M. Farenzena, L. Bazzani, A. Perina, V. Murino and M. Cristani, "Person re-identification by symmetry-driven accumulation of local features", In IEEE computer society conference on computer vision and pattern recognition, pp. 2360-2367, 2010.

7. D.S. Cheng, M. Cristani, M. Stoppa, L. Bazzani, and V. Murino, "Custom pictorial structures for re-identification", In Bmvc,Vol. 1, No. 2, p. 6, 2011.

8. A. Bhuiyan, A. Perina, and V. Murino, "Person re-identification by discriminatively selecting parts and features", In European Conference on Computer Vision, Springer, Cham, pp. 147-161, 2014.

9. I. Kviatkovsky, A. Adam, and E. Rivlin, "Color invariants for person re-identification", IEEE Transactions on pattern analysis and machine intelligence, 35(7), pp.1622-1634, 2012.

10. M. Hirzer, P.M. Roth, M. Köstinger and H. Bischof, "Relaxed pairwise learned metric for person re-identification", In European conference on computer vision, Springer, Berlin, Heidelberg, pp. 780-793, 2012.

11. A. Mignon and F. Jurie, "Pcca: A new approach for distance learning from sparse pairwise constraints", In IEEE conference on computer vision and pattern recognition, pp. 2666-2672, 2012.
12. S. Liao, Y. Hu, X. Zhu and S.Z. Li, "Person re-identification by loca maximal occurrence representation and metric learning", In Proceedings of the IEEE conference on computer vision and pattern recognition, pp. 2197-2206, 2015.

13. T. Matsukawa, T. Okabe, E. Suzuki and Sato, "Hierarchical gaussian descriptor for person re-identification", In Proceedings of the IEEE conference on computer vision and pattern recognition, pp. 1363-1372, 2016.

14. W.R. Schwartz, and L.S. Davis, "Learning discriminative appearance-based models using partial least squares", In XXII Brazilian symposium on computer graphics and image processing, pp. 322-329, 2009.

15. S. Bak, E. Corvee, F. Bremond and M. Thonnat, "Person re-identification using spatial covariance regions of human body parts", In IEEE International Conference on Advanced Video and Signal Based Surveillance, pp. 435-440, 2010.

16. B.J. Prosser, S. Gong and T. Xiang, "Multi-camera Matching using Bi-Directional Cumulative Brightness Transfer Functions", In BMVC, Vol. 8(164), p. 74, 2008.

17. M. Koestinger, M. Hirzer, P. Wohlhart, P.M. Roth and H. Bischof, "Large scale metric learning from equivalence constraints", In IEEE conference on computer vision and pattern recognition, pp. 2288-2295, 2012.

18. B. Schölkopf, A. Smola and K.R. Müller, "Nonlinear component analysis as a kernel eigenvalue problem", Neural computation, 10(5), pp.1299-1319, 1998.

19. K. Weinberger, B. Packer and L. Saul, "Nonlinear dimensionality reduction by semidefinite programming and kernel matrix factorization", In International Workshop on Artificial Intelligence and Statistics, pp. 381-388, 2005.

20. G. Lisanti, I. Masi, A.D. Bagdanov and A. Del Bimbo, "Person re-identification by iterative re-weighted sparse ranking", IEEE transactions on pattern analysis and machine intelligence, 37(8), pp.1629-1642, 2015.

21. L. Bazzani, M. Cristani and V. Murino, "Symmetry-driven accumulation of local features for human characterization and re-identification", Computer Vision and Image Understanding, 117(2), pp.130-144, 2013.

22. A. Das, A. Chakraborty and A.K. Roy-Chowdhury, "Consistent re-identification in a camera network", In European conference on computer vision, Springer, Cham, pp. 330-345, 2014.

23. A. Bhuiyan, B. Mirmahboub, A. Perina and V. Murino, "Person re-identification using robust brightness transfer functions based on multiple detections", In International Conference on Image Analysis and Processing, Springer, Cham, pp. 449-459, 2015.

24. Q. Wang, "Kernel principal component analysis and its applications in face recognition and active shape models", arXiv preprint arXiv:1207.3538, 2012.

\section{AUTHORS PROFILE}

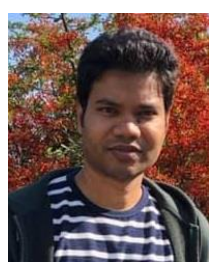

Md Kamal Uddin, is currently working as an Assistant Professor in the department of Computer Science and Telecommunication Engineering at Noakhali Science and Technology University, Noakhali, Bangladesh. His main research interests include computer vision, pattern recognition, machine learning, person re-identification and video surveillance techniques.

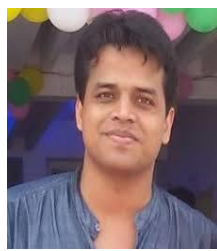

Amran Bhuiyan, received the Bachelor degree in Applied Physics, Electronic \& Communication Engineering from the University of Dhaka, Bangladesh in 2009, the M.Sc. degree in Computer Engineering and Information Technology from the Lucian Blaga University of Sibiu,Romania under the Erasmus Mundus external window in 2011 and the Ph.D. degree in Pattern Analysis and Computer Vision from the Istituto Italiano di Tecnologia, Genova, Italy. Previously, he was a Mitacs Elevate Postdoctoral Researcher with LIVIA, École de Technologie Supérieure, Université du Québec, Montréal, Canada and the industrial partner organization is SPORTLOGiQ INC. Currently, he is working as Associate Professor in the department of Computer Science and Telecommunication Engineering at Noakhali Science and Technology University, Bangladesh.

Published By:

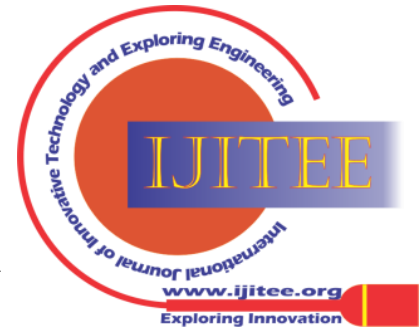




\section{Impact of Kernel-PCA on Different Features for Person Re-Identification}

His main research interests include computer vision, machine learning techniques for image and video processing, with applications such as video surveillance, summarizations, and sports analytics, and seeks to match or recognize individuals across non-overlapping views in a multi-camera system. Apart from these, he involves himself in designing simulation tools for the application of parameter variations in micro and macro electronic devices.

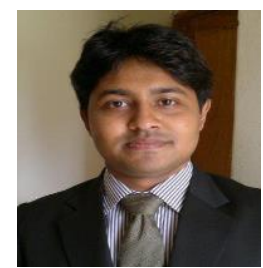

Mahmudul Hasan, is currently working as a computer science and engineering faculty member at Comilla University, Cumilla, Bangladesh. He obtained an M.Sc. (Thesis) in Computer Science and Engineering from the University of Rajshahi, Bangladesh. His research interest includes Machine Learning, Computer Vision and Human-Computer Interaction (HCI).

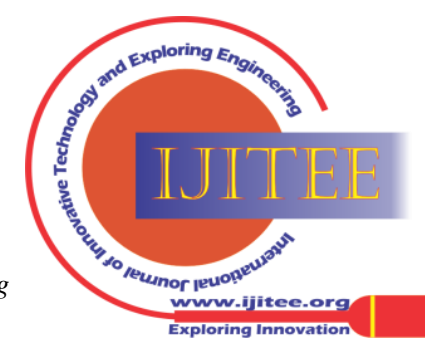

